\title{
An Evaluation of Ovarian Response and Pregnancy Rates with the Use of Growth Hormone as an Adjunct to IVF in Women who are Poor Responders to Standard IVF Stimulation Protocols
}

\author{
Mohamed T* and Cassim MI \\ BioART Fertility Centre, South Africa
}

*Corresponding author: Mohamed T, BioART fertility centre, Saxonwold, Johannesburg, South Africa.

\begin{abstract}
Background: Women who fail to respond adequately to standard ovarian stimulation protocols pose a significant treatment challenge. Research has been conducted in order to identify risk factors and causes of poor ovarian response and has attempted to identify new strategies which may improve the response to ovarian stimulation with standard IVF protocols. Growth hormone (GH) supplementation is one of these strategies proposed as a management option for poor IVF responders. GH is needed for growth and development but is also involved in the modulation of both male and female fertility through both gonadotrophin-dependent and gonadotrophin-independent actions. Some studies have shown that the supplementation of GH as an adjunct to ovarian stimulation for poor ovarian responders improves oocyte quality and increases pregnancy rates; while other studies has shown it to have no significant effect.
\end{abstract}

Objectives: To evaluate the effects of GH as an adjunct to IVF on ovarian response, oocyte quality and embryo grade as well as its effects on the achievement of pregnancy.

Methods: A retrospective cohort study of a subgroup of women attending a Fertility Centre in Johannesburg, who had responded poorly to stimulation protocols in previous IVF cycles. Those that had GH supplementation in addition to routine ovarian stimulation comprised the study group and those that did not were the controls.

Results: There were 98 women in the control and 103 in the study group. The mean age of the study group was older (38 vs 36 years) and the control group had higher AMH levels, especially amongst the respondents under the age of 40 . Both groups were statistically significantly different with regards to AMH levels and age, p-values 0.000 and 0.007 respectively. The two groups produced on average equal numbers of oocytes, embryos and embryos for ET.

The study group exhibited more pregnancies than the control group (35 vs 30) although this was not statistically significant ( $p$-value $>0.05$ ). The control group had on average, women of slightly younger age falling pregnant (35 vs 38 years). The study group had more respondents over the age of 40 years achieving pregnancy ( 14 vs 6). AMH levels were higher amongst women who achieved pregnancy in the control group (3.61 vs 2.78) but were only negligibly different for positive responders in the study group (1.57 vs 1.32). There was no statistically significant difference noted for the quality and quantity of the embryos for ET between the two groups.

Conclusion: This study suggests that GH is a useful adjunct in the treatment of women who are poor ovarian responders. It demonstrated that despite the fact that the study group had both on average an older age and lower AMH levels, they had significantly more pregnancies than expected for those under the age of 35 and relatively, although not statistically significantly more pregnancies than expected for those over the age of 35 (28\% vs $25 \%$ ).

Keywords: Growth hormone; IVF stimulation protocol; Ovarian response; Pregnancy 


\section{Background}

Women who fail to respond adequately to standard ovarian stimulation protocols pose a significant treatment challenge. Research has been conducted in order to identify risk factors and causes of poor ovarian response and has attempted to identify new strategies which may improve the response to ovarian stimulation with standard IVF protocols [1-3]. Growth hormone $(\mathrm{GH})$ supplementation is one of these strategies proposed as a management option for poor IVF responders. GH is needed for growth and development but is also involved in the modulation of both male and female fertility through both gonadotrophindependent and gonadotrophin-independent actions. GH has been shown to participate in gonadal steroidogenesis, gametogenesis and also has a role in ovulation [4-5]. Some studies have shown that the supplementation of GH as an adjunct to ovarian stimulation for poor ovarian responders improves oocyte quality and increases pregnancy rates [6-9]. While other studies and meta-analysis has shown it to have no significant effect [10-11].

\section{Objectives}

To evaluate the effects of GH as an adjunct to standard IVF protocols on ovarian response. The study looked at the influence of GH on oocyte quality and embryo grade as well as its effects on the achievement of pregnancy.

\section{Materials and Methods}

This was a retrospective cohort study. The records of 201 women were reviewed. All of these women had undergone an IVF treatment cycle in the preceding 12 months. Those who had received growth hormone treatment were included in the study group and those that had not comprised the control group. All those studied were women who were known poor responders and had responded poorly to ovarian stimulation in previous IVF cycle/s. There were 98 in the control group and 103 in the study group. The AMH (ng/mol) levels of both groups were measured and recorded prior to IVF treatment, as were there relation to the average for their age group. Other variables of record were age group and BHCG result (positive or negative). Further variables, the number of oocytes, embryos, embryos for ET were recorded post treatment. Data was collected on the quality and the grade of the embryos.

Ethics clearance for this study was sought from and granted by the DUT (Durban University of Technology) institutional review board. Written consent from the women who received growth hormone treatment was obtained prior to treatment.

The benefit of growth hormone supplementation is controversial and growth hormone has been used at BioART fertility centre since 2015. It is used as an adjunctive treatment to IVF therapy for consenting known poor responder patients to try and improve outcomes of ovarian stimulation. The purpose of the study was to evaluate the effects of the treatment in our own clinical setting and whether this treatment modality is indeed beneficial and if so, then for which patients.

\section{Results}

\section{Sample overview}

The sample found more pregnancies in the study than in the control condition (35 to 30). The average age of the study group was older (38 years to 36) and the control group had higher AMH (ng/mol) levels, especially among the respondents under the age of 40 . Both groups were statistically significantly different with regards to AMH (ng/mol) levels and age, with the treatment group being low in AMH (ng/mol) and high in age ( $p=0.000$ for AMH and $\mathrm{p}=0.007$ for age). AMH (ng/mol) levels were taken before treatment. The number of oocytes, embryos, embryos for ET, their grade and cell number were recorded retrospectively. These figures show that the two groups produced equal numbers, on average, of oocytes, embryos and embryos for ET. The control group produced proportionally more grade 1 embryos (64\%) than the study group (55\%) (Table 1).

Table 1: Sample overview - control group vs study group.

\begin{tabular}{|c|c|c|c|c|}
\hline & & & \multicolumn{2}{|c|}{ Source of Data } \\
\hline & & & Control & Study \\
\hline \multirow[t]{2}{*}{ BHCG_result } & Negative & Count & 68 & 68 \\
\hline & Positive & Count & 30 & 35 \\
\hline \multirow[t]{2}{*}{ AGE } & Mean & & 36.1 & 38.1 \\
\hline & Standard Deviation & & 5.5 & 4.4 \\
\hline \multirow[t]{2}{*}{ Age_grouped } & Under 40 & AMH (ng/mol) & 3.06 & 1.53 \\
\hline & Over 40 & $\mathrm{AMH}(\mathrm{ng} / \mathrm{mol})$ & 1.36 & 1.17 \\
\hline \multirow[t]{2}{*}{ AMH (ng/mol) } & Mean & & 2.62 & 1.4 \\
\hline & Standard Deviation & & 2.72 & 1.16 \\
\hline \multirow[t]{2}{*}{ AMH (ng/mol) level relative to average } & AMH below average & Count & 49 & 72 \\
\hline & AMH greater than & Count & 49 & 29 \\
\hline No of Oocytes & Mean & Count & 8 & 8 \\
\hline No of Embryos & Mean & Count & 5 & 5 \\
\hline Embryos for ET & Mean & Count & 3 & 3 \\
\hline Grade 1 Number & Mean & Count & 2 & 2 \\
\hline Grade 1_percentage/proportion & Mean & Count & $64 \%$ & $55 \%$ \\
\hline
\end{tabular}




\begin{tabular}{|c|c|c|c|c|}
\hline Grade 2 Number & Mean & Count & 1 & 1 \\
\hline Grade 2 Percentage & Mean & Count & $31 \%$ & $33 \%$ \\
\hline Grade 1 Cell ( 6 or more cells) & Mean & Count & 1.12 & 0.7 \\
\hline Grade 2 Cell ( 6 or more cells) & Mean & Count & $59 \%$ & $74 \%$ \\
\hline Grade 1 Cell ( 5 or fewer) & Mean & Count & 0.16 & 0.21 \\
\hline Grade 2 Cell ( 5 or fewer) & Mean & Count & $15 \%$ & $14 \%$ \\
\hline
\end{tabular}

A review of the sample by study/control and pregnancy (positive/negative) demonstrates that the control group had on average, women of slightly younger age falling pregnant (35 years versus 37 ) but this was not the case for the study group, where the average age was 38 for both conditions. The study group had far more respondents over 40 and positive for pregnancy (14 versus 6). The AMH (ng/mol) figures were far higher for respondents who tested positive in the control condition (3.61 versus 2.78 ) but negligibly different for positive respondents in the study condition
(1.57 versus 1.32) (Figure 1). Interestingly, number of oocytes was higher in the negative response across both the study and the control group, as were the number of embryos. The number of embryos for ET was on average equal across all conditions and results, as was the number of grade 1 embryos. Hence there were no statistically significant difference between the quantity and quality of embryos of the two groups. These figures were recorded post hoc and reflect therefore potentially the impact of the treatment itself (Table 2).

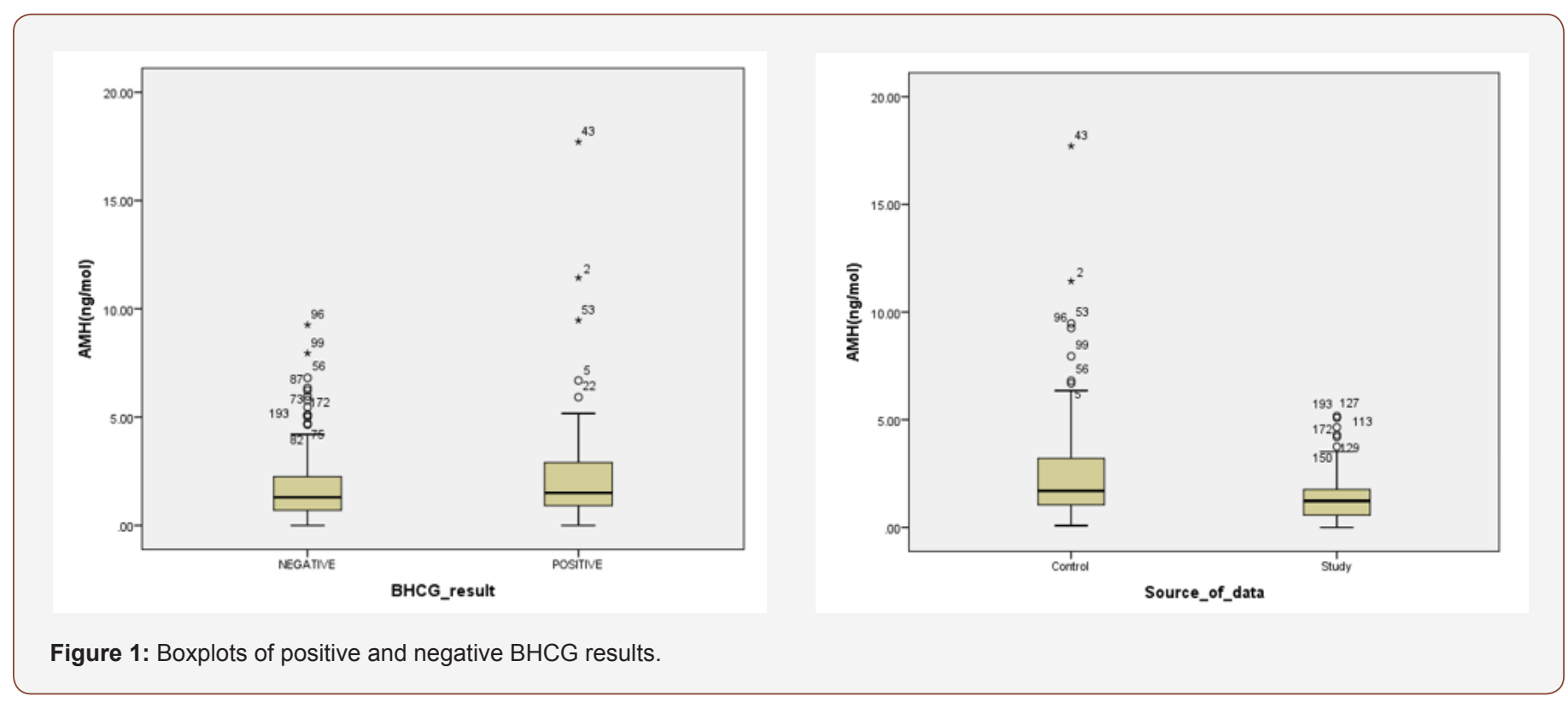

Table 2: Overview of sample (study/control) and pregnancy outcome.

\begin{tabular}{|c|c|c|c|c|c|c|}
\hline & & & \multicolumn{4}{|c|}{ Source of Data } \\
\hline & & & \multirow{2}{*}{\multicolumn{2}{|c|}{$\begin{array}{c}\text { Control } \\
\text { BHCG_result }\end{array}$}} & \multirow{2}{*}{\multicolumn{2}{|c|}{$\begin{array}{c}\text { Study } \\
\text { BHCG_result }\end{array}$}} \\
\hline & & & & & & \\
\hline & & & Negative & Positive & Negative & Positive \\
\hline \multirow[t]{2}{*}{ Age } & Mean & & 36.7 & 34.8 & 38 & 38.1 \\
\hline & Standard Deviation & & 5.6 & 5.3 & 3.9 & 5.3 \\
\hline \multirow[t]{2}{*}{ Age_Grouped } & Under 40 & Count & 49 & 24 & 44 & 21 \\
\hline & Over 40 & Count & 19 & 6 & 24 & 14 \\
\hline \multirow[t]{2}{*}{ Age_Grouped } & Under 40 & AMH (ng/mol) Mean & 2.78 & 3.61 & 1.33 & 1.95 \\
\hline & Over 40 & AMH (ng/mol) Mean & 1.27 & 1.65 & 1.29 & 0.95 \\
\hline \multirow[t]{2}{*}{ AMH (ng/mol) } & Mean & & 2.36 & 3.22 & 1.32 & 1.57 \\
\hline & Standard Deviation & & 2.04 & 3.81 & 1.11 & 1.26 \\
\hline \multirow[t]{2}{*}{$\begin{array}{c}\text { AMH ( } \mathrm{ng} / \mathrm{mol} \text { ) level relative to } \\
\text { average }\end{array}$} & AMH below average & Count & 35 & 14 & 50 & 22 \\
\hline & AMH greater than average & Count & 33 & 16 & 17 & 12 \\
\hline \multirow[t]{2}{*}{ No of oocytes } & Mean & Count & 9 & 7 & 8 & 7 \\
\hline & Standard Deviation & Count & 6 & 4 & 5 & 4 \\
\hline
\end{tabular}




\begin{tabular}{|c|c|c|c|c|c|c|}
\hline No of embryos & Mean & Count & 5 & 4 & 6 & \\
\hline & Standard Deviation & Count & 3 & 2 & 4 & \\
\hline \multirow[t]{2}{*}{ Embryos for ET } & Mean & Count & 3 & 3 & 3 & \\
\hline & Standard Deviation & Count & 1 & 1 & 1 & \\
\hline \multirow[t]{2}{*}{ Grade 1 Number } & Mean & Count & 2 & 2 & 2 & \\
\hline & Standard Deviation & Count & 1 & 1 & 1 & \\
\hline \multirow[t]{2}{*}{ Grade 1_percentage/proportion } & Mean & Count & $64 \%$ & $65 \%$ & $55 \%$ & $54 \%$ \\
\hline & Standard Deviation & Count & $36 \%$ & $26 \%$ & $40 \%$ & $40 \%$ \\
\hline \multirow[t]{2}{*}{ Grade 2 Number } & Mean & Count & 1 & 1 & 1 & 1 \\
\hline & Standard Deviation & Count & 1 & 1 & 1 & 1 \\
\hline \multirow[t]{2}{*}{ Grade 2_percentage } & Mean & Count & $33 \%$ & $27 \%$ & $33 \%$ & $33 \%$ \\
\hline & Standard Deviation & Count & $36 \%$ & $28 \%$ & $35 \%$ & $36 \%$ \\
\hline \multirow[t]{2}{*}{ Grade 1 cell ( 6 or more cells) } & Mean & Count & 1.14 & 1.08 & $67 \%$ & $76 \%$ \\
\hline & Standard Deviation & Count & 1.31 & 1.13 & $91 \%$ & $1 \%$ \\
\hline \multirow[t]{2}{*}{ Grade 1 cell ( 5 or fewer) } & Mean & Count & 0.14 & 0.19 & $29 \%$ & $7 \%$ \\
\hline & Standard Deviation & Count & 0.44 & 0.57 & $97 \%$ & $26 \%$ \\
\hline \multirow[t]{2}{*}{ Grade 2 cell ( 5 or fewer) } & Mean & Count & 0.14 & 0.15 & $12 \%$ & $17 \%$ \\
\hline & Standard Deviation & Count & 0.4 & 0.46 & $33 \%$ & $47 \%$ \\
\hline
\end{tabular}

\section{Assumptions checking: AMH (ng/mol) level}

AMH (ng/mol) levels were not normally distributed, whether for the control or the study groups or overall. All three distributions were positively skewed, and the Kolmogorov-Smirnov and Shapiro Wilk tests were significant $(p=0.000)$. The skewness $(3.198)$ is above three times the error $(0.172)$ at 18 times the standard error. There were also a number of outliers.

When the trimmed mean is compared to the mean, however, the results are fairly close $(1.83 \mathrm{ng} / \mathrm{mol}$ versus $1.65 \mathrm{ng} / \mathrm{mol}$ for the "Negative" distribution and $1.9 \mathrm{ng} / \mathrm{mol}$ versus $2.3 \mathrm{ng} / \mathrm{mol}$ for the "Positive" distribution), suggesting these outliers may have less of an impact. Between the study and experimental groups, the difference between the trimmed means and the mean was $1.2 \mathrm{ng} /$ mol and $1.4 \mathrm{ng} / \mathrm{mol}$ and $2.2 \mathrm{ng} / \mathrm{mol}$ and $2.6 \mathrm{ng} / \mathrm{mol}$ respectively. The lower difference between trimmed and actual means indicates that the outliers may not be of great concern.

Table 3: Tests of normality for the transformed AMH ng/mol variable.
The Levene's test revealed that the variance for the study/ control group as well as the BCHG positive/negative groups was heteroscedastic ( $\mathrm{p}=0.000$ for both comparisons). Furthermore, the difference between women over 40 and under 40 also had $p=0.001$ on Levene's test. Due to the lack of homoscedasticity, the large degree of negative skewness present, AMH ng/mol was transformed by taking a $\log 10$ of the values. The subsequent Levene's tests were no longer significant, with $p=0.396$ between the control and the study group and $p=0.729$ between the women who had positive and negative BCHG results and $p=0.971$ for the difference between women of different age groups. Thus, the condition of homoscedasticity is now met. Unfortunately, the Kolmogorov-Smirnov and Shapiro Wilk tests were still significant for the transformed variables (the range of was $\mathrm{p}=0.01$ to $\mathrm{p}=$ 0.072) (Table 3).

\begin{tabular}{|c|c|c|c|c|c|c|c|}
\hline \multicolumn{8}{|c|}{ Test of Normality } \\
\hline & & Kolmogorov-Smirnov & & & Shapiro-Wilk & & \\
\hline & & Statistic & df & Sig. & Statistic & df & Sig. \\
\hline \multicolumn{8}{|l|}{ Source of Data } \\
\hline \multirow[t]{2}{*}{ LogAMH } & Control & 0.097 & 99 & 0.022 & 0.968 & 99 & 0.017 \\
\hline & Study & 0.016 & 99 & 0.008 & 0.953 & 99 & 0.001 \\
\hline \multirow[t]{2}{*}{ BHCG_result } & & Kolmogorov-Smirnov ${ }^{\mathrm{a}}$ & & & Shapiro-Wilk & & \\
\hline & & Statistic & $\mathrm{df}$ & Sig & Statistic & df & Sig \\
\hline \multirow[t]{3}{*}{ LogAMH } & Negative BCHG & 0.088 & 135 & 0.011 & 0.964 & 135 & 0.001 \\
\hline & Positive BCHG & 0.107 & 63 & 0.072 & 0.974 & 63 & 0.203 \\
\hline & & Kolmogorov-Smirnov $_{a}$ & & & Shapiro-Wilk & & \\
\hline \multirow[t]{3}{*}{ Age Grouped } & & Statistic & $\mathrm{df}$ & Sig & Statistic & df & Sig \\
\hline & Under 40 & 0.075 & 139 & 0.055 & 0.973 & 139 & 0.007 \\
\hline & Over 40 & 0.146 & 59 & 0.003 & 0.954 & 59 & 0.026 \\
\hline
\end{tabular}

aLilliefors significance Correction 
A review of the transformed box plot (Figure 2) as well as the normal Q-Q plots for the AMH ng/mol and its transformed value (Figures 3-5) indicates that outliers \#43, 162, 25 and 58 could remain problematic. The difference between trimmed mean and means remained small. The histogram demonstrates a vast improvement in distribution and the degree of skewness is greatly reduced. The skewness is less than the advised maximum of three times the standard error and is only 2.2 times (skewness $=-.388$ and the standard error is .173). In order to deal with the potential import of outliers, scores which were higher than three standard deviations from the mean were excluded from the analysis. The AMH (ng/mol) levels of the affected patients were 7.29540; 4.37825; 3.46780 and 3.37025 standard deviations from the mean respectively.
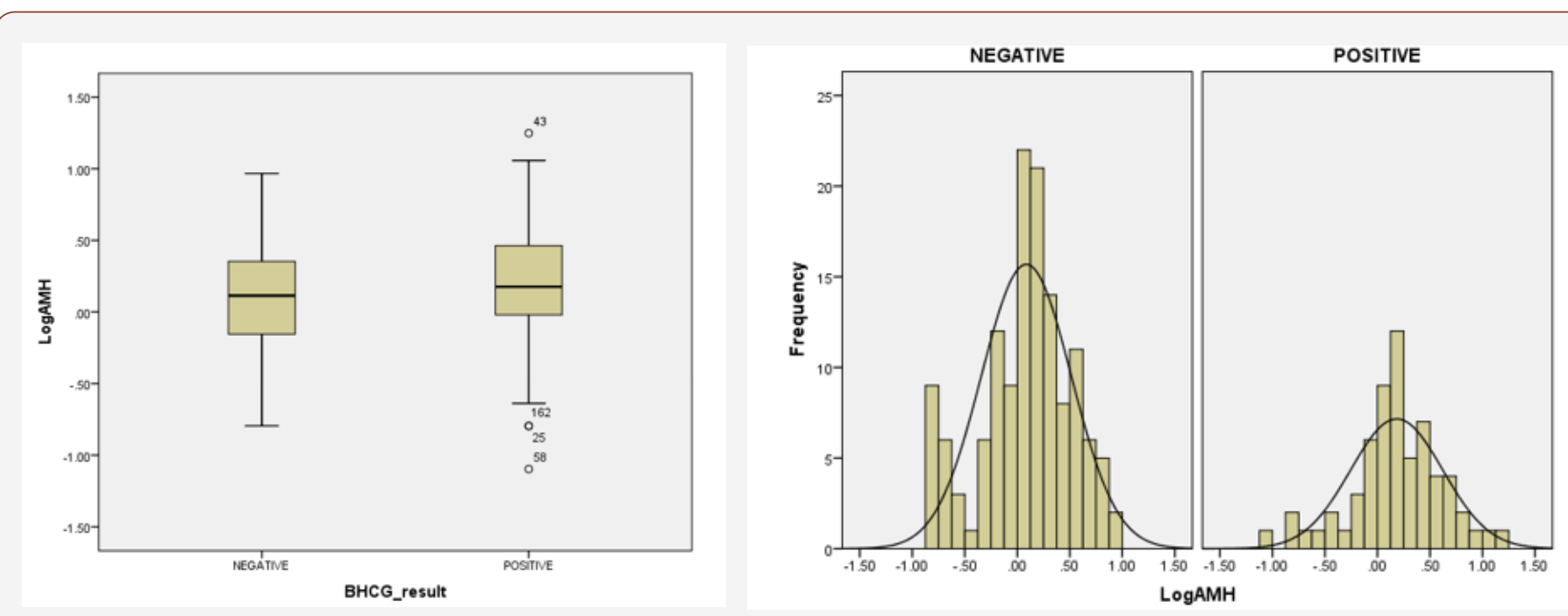

Figure 2: Boxplot and histogram of transformed $\mathrm{AMH} \mathrm{ng} / \mathrm{mol}$.
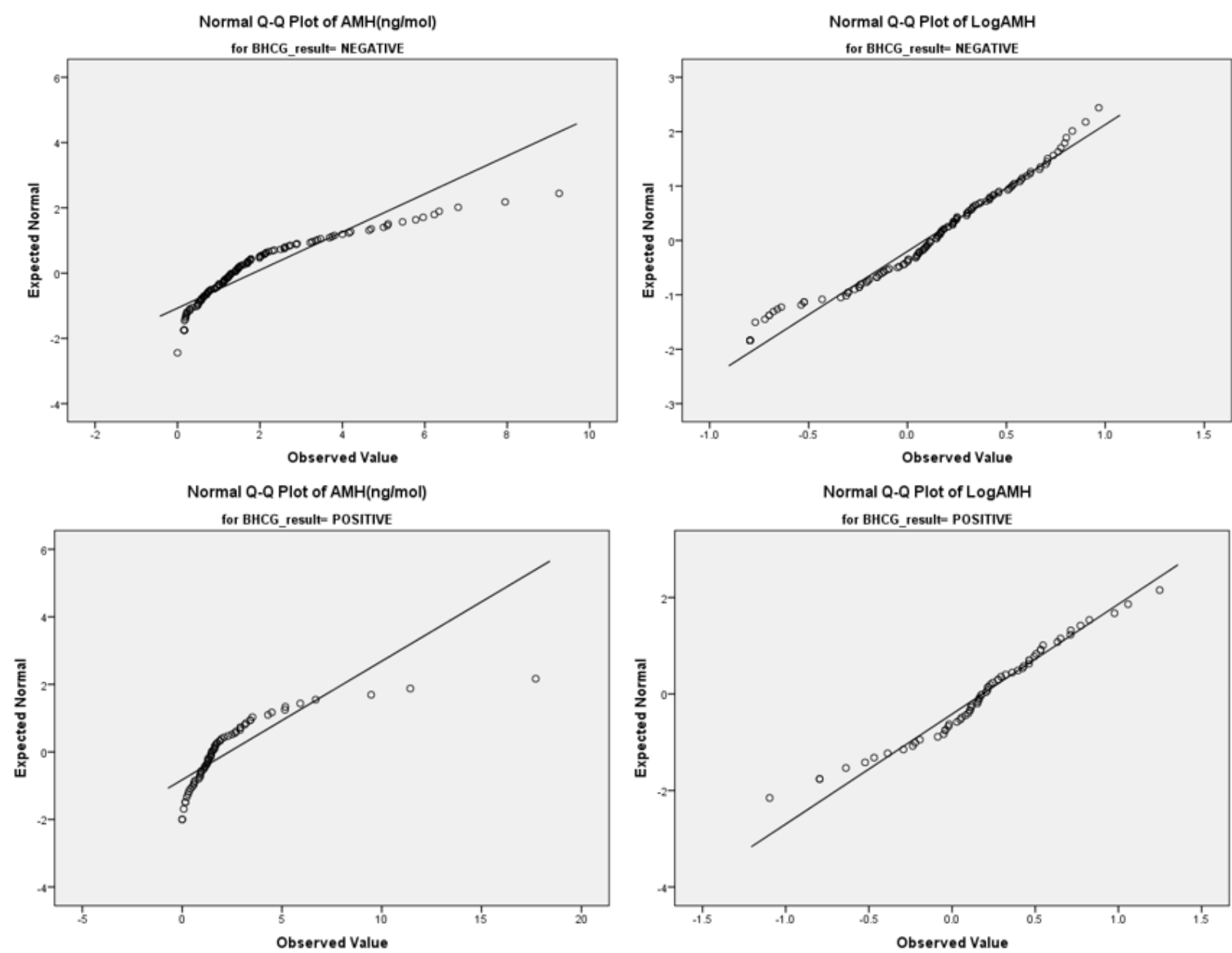

Figure 3: Normal Q-Q plots of $\mathrm{AMH} n \mathrm{ng} / \mathrm{mol}$ before and after log10 transformation for $\mathrm{BCHG}$ result. 

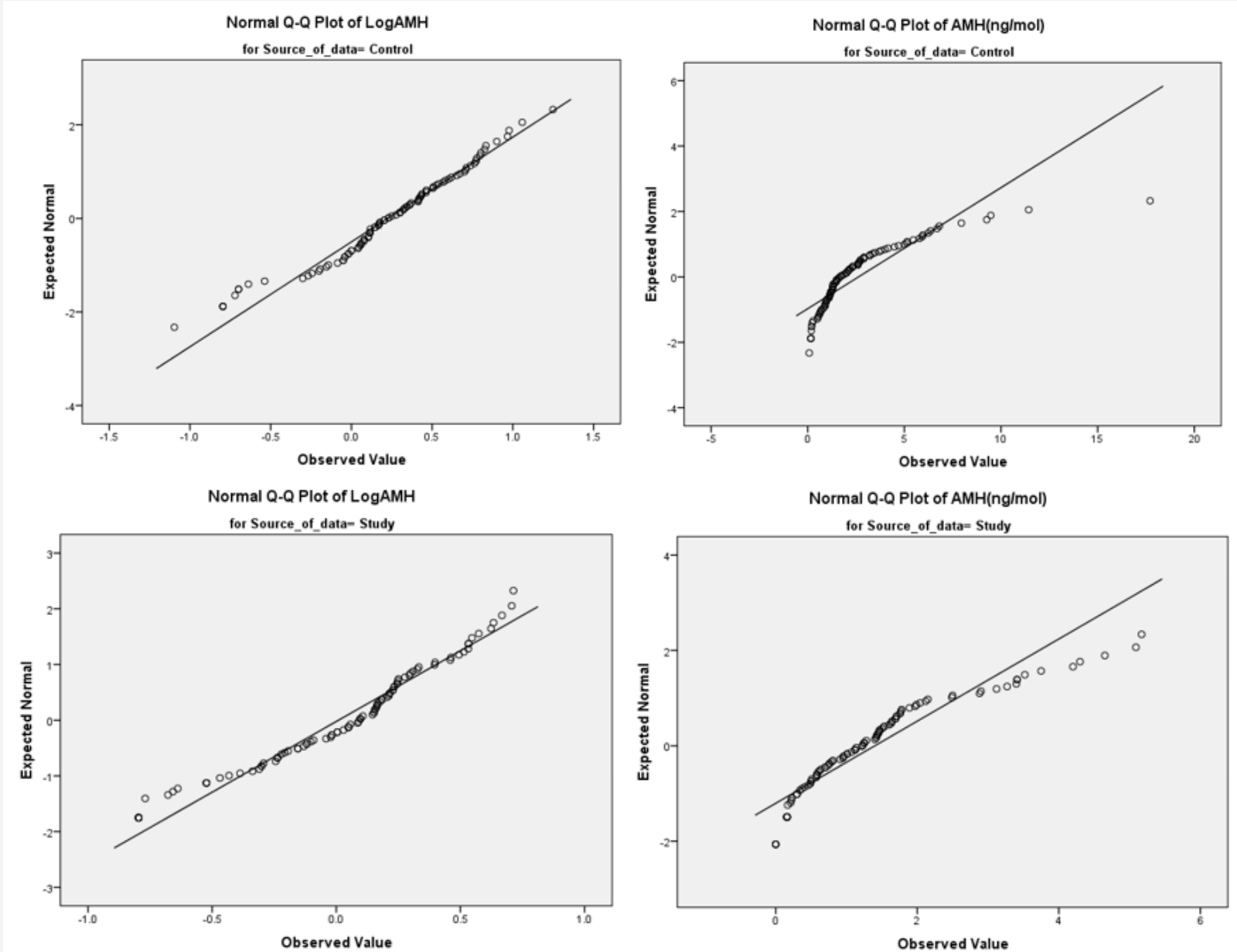

Figure 4: Normal Q-Q plots of AMH ng/mol before and after log10 transformation for control and study groups.
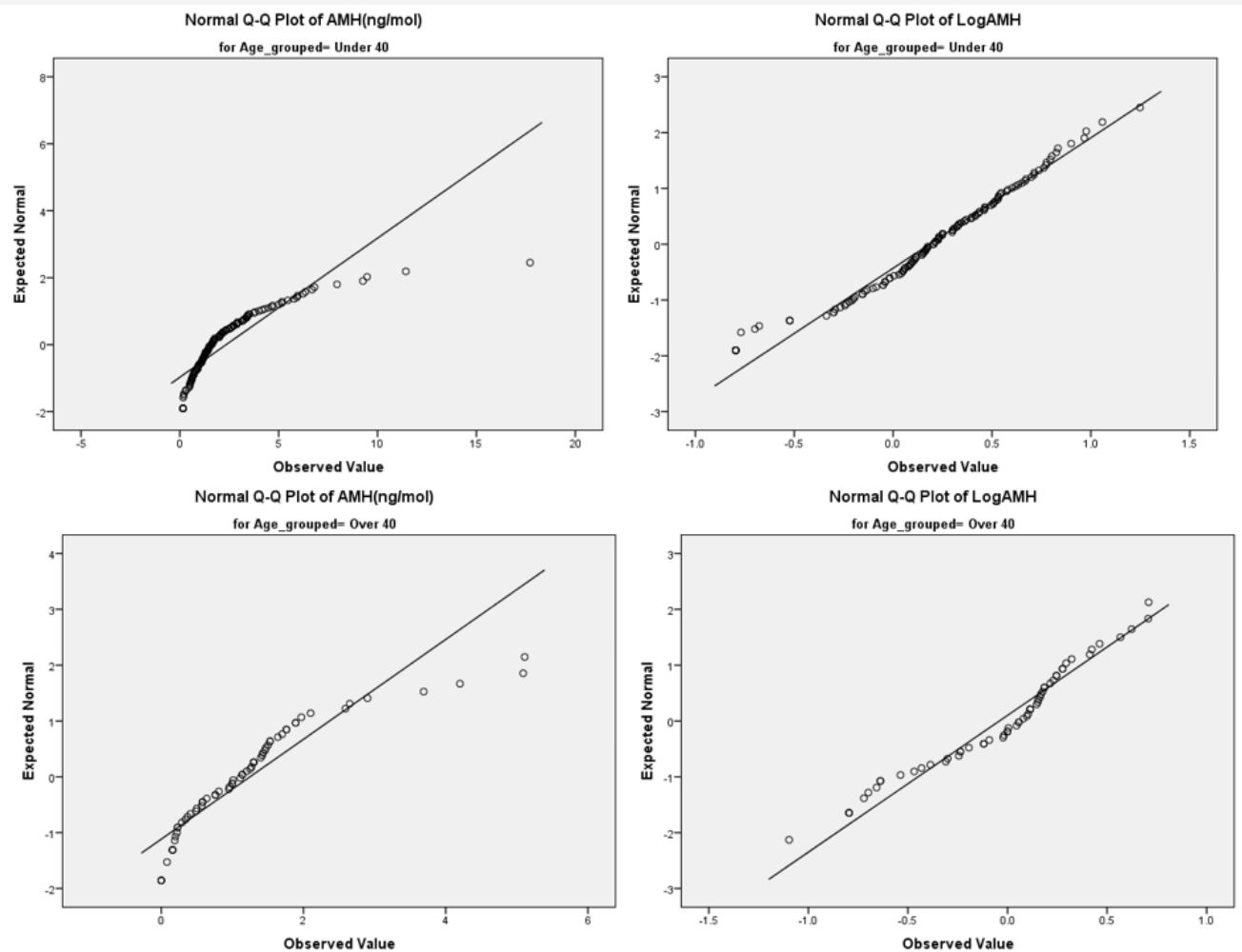

Figure 5: Normal Q-Q plots of AMH ng/mol before and after log10 transformation for over and under 40 age group. 


\section{Assumptions checking: Other variables}

Before these variables could be entered into the equation, the assumptions behind the remaining variables: Number of oocytes, Number of embryos, Number of embryos for ET, Embryo grade and Number of cells were checked. Cell numbers per grade were included and this was grouped into "Number of embryos with six or more cells" and "Number of embryos with five or fewer cells." The percentage for each grade (for example "Percentage of grade 1 cells") was calculated. Both the Kolmogorov-Smirnov and the Shapiro-Wilk tests were significant for all variables, necessitating transformation. The following variables were found to be excessively skewed across both groups: number of oocytes, number of embryos and number of embryos with 6 or more cells. In the study, rather than the control, the number of embryos with five or fewer cells had skewness figures greater than three times the standard error (Table 4).

Table 4: Tests of normality.

\begin{tabular}{|c|c|c|c|c|c|c|c|}
\hline \multicolumn{8}{|c|}{ Tests of Normality } \\
\hline & \multirow{2}{*}{ Source of Data } & \multicolumn{3}{|c|}{ Kolmogorov-Smirnova } & \multicolumn{3}{|c|}{ Shapiro-Wilk } \\
\hline & & Statistic & $\mathrm{df}$ & Sig. & Statistic & $\mathrm{df}$ & Sig. \\
\hline \multirow[t]{9}{*}{ Control } & No of Oocytes & 0.187 & 82 & 0 & 0.905 & 82 & 0 \\
\hline & No of Embryos & 0.206 & 82 & 0 & 0.896 & 82 & 0 \\
\hline & Embryos for ET & 0.203 & 82 & 0 & 0.912 & 82 & 0 \\
\hline & Grade 1 Number & 0.219 & 82 & 0 & 0.88 & 82 & 0 \\
\hline & Grade 1_percentage/proportion & 0.181 & 82 & 0 & 0.873 & 82 & 0 \\
\hline & Grade 2 Number & 0.255 & 82 & 0 & 0.814 & 82 & 0 \\
\hline & Grade 2 percentage & 0.254 & 82 & 0 & 0.83 & 82 & 0 \\
\hline & Grade 1 cell ( 6 or more cells) & 0.303 & 82 & 0 & 0.784 & 82 & 0 \\
\hline & Grade 2 cell ( 6 or more cells) & 0.35 & 82 & 0 & 0.732 & 82 & 0 \\
\hline \multirow[t]{9}{*}{ Study } & No of Oocytes & 0.124 & 80 & 0.004 & 0.91 & 80 & 0 \\
\hline & No of Embryos & 0.185 & 80 & 0 & 0.839 & 80 & 0 \\
\hline & Embryos for ET & 0.191 & 80 & 0 & 0.922 & 80 & 0 \\
\hline & Grade 1 Number & 0.178 & 80 & 0 & 0.902 & 80 & 0 \\
\hline & Grade 1_percentage/proportion & 0.21 & 80 & 0 & 0.838 & 80 & 0 \\
\hline & Grade 2 Number & 0.249 & 80 & 0 & 0.826 & 80 & 0 \\
\hline & Grade 2 percentage & 0.251 & 80 & 0 & 0.829 & 80 & 0 \\
\hline & Grade 1 cell ( 6 or more cells) & 0.358 & 80 & 0 & 0.726 & 80 & 0 \\
\hline & Grade 2 cell ( 6 or more cells) & 0.366 & 80 & 0 & 0.711 & 80 & 0 \\
\hline
\end{tabular}

aLilliefors significance Correction

When the trimmed mean is compared to the mean, however, the results are fairly close, suggesting that outliers may have less of an impact. Homoscedasticity was ascertained to hold for all variables bar number of grade 1 embryos and number of grade 1 cells ( 6 or more). Due to the skewness and normality issues for all bar number of embryos for transfer, several transformations were undertaken from the moderate (a square root of the number) to severe $(\log 10$ of the values and -1 divided by the square root of the values). None of these variables reflected much improvement in the normality tests $p<0.001$, part from number of oocytes ( $p=0.09$ ). Nevertheless, when the Q-Q plots were reviewed, most variables, apart from the percentage of grade 1 and percentage of grade 1 , tracked closely to the line. In terms of skewness, all variables apart from "Percentage of grade 1" had skewness results below that of three times their standard error. On this basis it was concluded that the severe transformed variables be used with the omission of the proportional percentages (percentage of grade 1 and 2) (Table 5).

Table 5: Descriptive statistics.

\begin{tabular}{|c|c|c|c|c|c|c|c|c|}
\hline \multicolumn{2}{|c|}{ Source of Data } & $\mathrm{N}$ & Minimum & Maximum & Mean & Std Deviation & \multicolumn{2}{|c|}{ Skewness } \\
\cline { 3 - 10 } & & Statistic & Statistic & Statistic & Statistic & Statistic & Statistic & Std error \\
\hline Control & No of Oocytes & 82 & 1 & 24 & 8.39 & 5.409 & 1.050 & 0.266 \\
\hline & No of Embryos & 82 & 1 & 15 & 4.80 & 3.053 & 1.134 & 0.266 \\
\hline & Embryos for ET & 82 & 1 & 5 & 3.01 & 1.094 & -.025 & 0.266 \\
\hline & Grade 1 Number & 82 & 0 & 4 & 2.01 & 1.094 & -.0430 & 0.266 \\
\hline & Grade 1_percentage/proportion & 82 & $0.00 \%$ & $100.00 \%$ & $64.1667 \%$ & $32.67797 \%$ & -.629 & 0.266 \\
\hline & Grade 1 cell (6 or more cells) & 82 & 0.00 & 4.00 & 1.1220 & 1.25108 & 0.539 & 0.266 \\
\hline & Grade 2 cell (6 or more cells) & 82 & 0.00 & 3.00 & 0.5854 & 0.76902 & 1.042 & 0.266 \\
\hline
\end{tabular}




\begin{tabular}{|c|c|c|c|c|c|c|c|c|}
\hline & Valid N (listwise) & 82 & & & & & \\
\hline Study & No of Oocytes & 80 & 1 & 28 & 7.59 & 4.906 & 1.284 & 0.269 \\
\hline & No of Embryos & 80 & 1 & 20 & 5.29 & 4.013 & 1.612 & 0.269 \\
\hline & Embryos for ET & 80 & 0 & 5 & 2.99 & 1.336 & -.238 & 0.269 \\
\hline & Grade 1 Number & 80 & 0 & 5 & 1.6 & 1.228 & .267 & 0.269 \\
\hline & Grade 1_percentage/proportion & 80 & $0.00 \%$ & $100.00 \%$ & $54.6875 \%$ & $39.82389 \%$ & -.171 & 0.269 \\
\hline & Grade 1 cell (6 or more cells) & 80 & 0 & 3.00 & 0.7000 & 0.94668 & 1.008 & 0.269 \\
\hline & Grade 2 cell (6 or more cells) & 80 & 0 & 4.00 & 0.7375 & 1.07614 & 1.297 & 0.269 \\
\hline & Valid N (listwise) & 80 & & & & & & \\
\hline
\end{tabular}

Once the variables were transformed, the file was split, and comparative analyses were performed to ascertain whether there was a predictive difference between the study and the control group. The model contained the following independent variables: AMH (ng/mol) level relative to average(1); AMH (ng/mol) (log of the values), No. oocytes (s. transformed), No. Embryos (s. transformed), No. Embryos for ET (s. transformed), No. Grade 1 Embryos (s. transformed), No. Grade 2 Embryos (s. transformed), and No. Embryos Grade 1 (6 or more cells) (s. transformed) and age.

\section{Discussion}

Linear discriminant analysis was used to examine if the possibility of conception could be predicted on the basis of AMH (ng/mol) levels and their increase through injection. Since these levels were assessed at the outset rather than subsequent to $\mathrm{AMH}$

Table 6: Results of discriminant analysis for control group.

\begin{tabular}{|c|c|c|c|c|c|}
\hline \multicolumn{6}{|c|}{ Classification Results $^{\mathrm{a}, \mathrm{c}}$} \\
\hline \multirow{2}{*}{\multicolumn{3}{|c|}{ BHCG_result }} & \multicolumn{3}{|c|}{ Predicted Group Membership } \\
\hline & & & Negative & Positive & Total \\
\hline \multirow{4}{*}{ Original } & \multirow{2}{*}{ Count } & Negative & 66 & 0 & 66 \\
\hline & & Positive & 28 & 0 & 28 \\
\hline & \multirow{2}{*}{$\%$} & Negative & 100.0 & .0 & 100 \\
\hline & & Positive & 100.0 & .0 & 100 \\
\hline \multirow{4}{*}{ Cross-validated $^{\mathrm{b}}$} & \multirow{2}{*}{ Count } & Negative & 66 & 0 & 66 \\
\hline & & Positive & 28 & 0 & 28 \\
\hline & \multirow{2}{*}{$\%$} & Negative & 100.0 & .0 & 100.0 \\
\hline & & Positive & 100.0 & .0 & 100.0 \\
\hline
\end{tabular}

a.

$70.2 \%$ of original grouped cases correctly classified.

b. Cross validation is done only for those cases in the analysis. In cross validation, each case is classified by the functions derived from all cases other than that case.

$70.2 \%$ of cross validated grouped cases correctly classified.

An initial binary logistic regression was then run on the control group data. The Homer-Lemeshow test was significant for the model which looked at AMH (ng/mol) levels and an age binary (over and under 40$)(p=0.05)$ meaning that the model did not fit the data. The model was re-run with age grouped into above and below 35 years. The second model had a Homer-Lemeshow $\mathrm{p}=$ 0.456, suggesting that the model fit the data. Nevertheless, none of the parameters entered, whether Age (binary) or AMH had significance in the model (both $\mathrm{p}>0.05$ ). Thus AMH (ng/mol) was not associated with pregnancy likelihood for the control group. We then moved onto a comparison of the control group pregnancy (ng/mol) intervention in the experimental group, there was no direct measure of AMH ( $\mathrm{ng} / \mathrm{mol}$ ) level in the experimental group prior to BCHG result. Therefore, the control group was used as a means to estimate probability for women who did not undergo treatment to generate the predictive possibility of the experimental group. The probability of the model between predicted and actual conception rates could be assessed. The intervention could be credited as a success if the experimental group outperformed expectations. Analysis was conducted in IBM SPSS.

The use of leave-one-out, cross-validated linear discriminant analysis, positive BHCG was not predicted for any of the patients and the classification table was able to correctly classify $70.2 \%$ of cases correctly, with complete misclassification for all positive pregnancies. The analysis was predicated on calculating prior probabilities based on the data itself, as no a priori figures were available (Table 6). outcome with that of the experimental group, whose AMH (ng/ mol) levels were stimulated through intervention by Norditropin injections.

There are significantly more pregnancies in the greater AMH than average and in the experimental condition (35) than expected ( $p=0.022 ; \chi=5.274$ ), which provides an effect size of $r=0.2263$; 95\% with a C.I. $=0.0337$ and 0.4026 ; Fisher's $\mathrm{Zr}=0.2303$ with a CI of 0.0337 and $0.4268 ; \mathrm{v}=0.0101$. Thus, the effect size is between small and medium (Hair, 2012) and is based on the sample size of the contingency table of the experiment. The sample size is too 
small to compare the age difference for women over the age of 40 to the others and thus an age division of over and less than 35 years was used. The role of AMH (ng/mol) was recorded based on levels prior to the intervention. AMH $(\mathrm{ng} / \mathrm{mol})$ was recorded as either above or below acceptable levels for the respective age group of each patient. Thus, when viewing the tables in question [Table 3], it is apparent that there were proportionally more pregnancies in the study group (35 versus 28) although this was not significant at $\mathrm{p}<0.05(\mathrm{p}=0.496)$. When the data is reviewed at the age level, the profile for positive BHCG is significantly different for women under 35 as $54.5 \%$ fell pregnant as opposed to $37 \%$ in the control condition ( $p=0.022)$. There is an absolute although not significant difference for women over 35 (28\% were pregnant as opposed to $25 \%$ in the control condition) [Table 7].

Table 7: Chi-square results by experimental condition and age.

\begin{tabular}{|c|c|c|c|c|c|c|c|c|}
\hline \multicolumn{9}{|c|}{ Source of Data } \\
\hline & & & \multicolumn{3}{|c|}{ Control } & \multicolumn{3}{|c|}{ Study } \\
\hline & \multicolumn{3}{|c|}{ Age Grouped 35} & \multicolumn{3}{|c|}{ Age Grouped 35} & & \\
\hline & Total & Under 35 & Over 35 & Total & Under 35 & Over 35 & & \\
\hline \multirow[t]{4}{*}{ BHCG result } & Negative & Count & 67 & 22 & 45 & 68 & 10 & 25 \\
\hline & & Column N\% & $70.5 \%$ & $62.9 \%$ & $75.0 \%$ & $66.0 \%$ & $45.50 \%$ & $71.6 \%$ \\
\hline & Positive & Count & 28 & 13 & 15 & 35 & 12 & 23 \\
\hline & & Column N\% & $29.5 \%$ & $37.1 \%$ & $25.0 \%$ & $34.0 \%$ & $54.5 \%$ & $28.4 \%$ \\
\hline \multicolumn{9}{|c|}{ Pearson Chi-Square Test } \\
\hline & \multicolumn{8}{|c|}{ Source of data } \\
\hline & \multicolumn{2}{|c|}{ Control } & \multicolumn{6}{|c|}{ Study } \\
\hline & \multicolumn{2}{|c|}{ Age Grouped } & \multicolumn{6}{|c|}{ Age Grouped } \\
\hline & \multicolumn{2}{|c|}{35} & \multicolumn{6}{|c|}{35} \\
\hline \multirow[t]{3}{*}{ BHCG result } & Chi square & 1.568 & & 5.274 & & & & \\
\hline & $\mathrm{df}$ & 1 & & 1 & & & & \\
\hline & Sig. & 0.21 & & $0.22^{*}$ & & & & \\
\hline \multicolumn{9}{|c|}{ Results are based on nonempty rows and columns in each innermost subtable } \\
\hline \multicolumn{9}{|c|}{ *The Chi square statistic is significant at the 0.05 level } \\
\hline \multicolumn{9}{|c|}{ Comparisons of Column Proportions ${ }^{a}$} \\
\hline & \multicolumn{8}{|c|}{ Source of data } \\
\hline & \multicolumn{3}{|c|}{ Control } & \multicolumn{5}{|c|}{ Study } \\
\hline & \multicolumn{3}{|c|}{ Age Grouped 35} & \multicolumn{5}{|c|}{ Age Grouped 35} \\
\hline & Under 35 & Over & & Under 35 & & & & \\
\hline & (A) & (B & & $(\mathrm{A})$ & & & & \\
\hline \multirow[t]{2}{*}{ BHCG result } & Negative & \multicolumn{7}{|c|}{$\mathrm{A}$} \\
\hline & Positive & \multicolumn{7}{|c|}{ B } \\
\hline Results are based c & tests with si & $\begin{array}{l}\text { cance level } .05 \text {. } \\
\text { s under the cat }\end{array}$ & $\begin{array}{l}\text { pr each sig } \\
\text { ory with tl }\end{array}$ & $\begin{array}{l}\text { ficant pair, th } \\
\text { larger colum }\end{array}$ & $\begin{array}{l}\text { key of the ca } \\
\text { proportion. }\end{array}$ & ry with sm & column $\mathrm{p}$ & tion ap \\
\hline
\end{tabular}

\section{Predictive power of variables in the model}

Since the evidence from the above analyses suggests that there is a heightened probability of the women in the experimental condition to fall pregnant but the binary variables of age (over and under 35) and AMH (ng/mol) (above and below average), had limited statistically significant findings. A wider set of variables were employed along with the use of actual age (a continuous variable) and actual AMH (ng/mol) (a continuous variable). The role of all variables on the BHCG result was ascertained by means of a hierarchical binary logistic regression. The dependent variable was the binary BHCG result and the independent variables were grouped as follows: Block 1 - AMH (ng/mol) level relative to average for the woman's age group; AMH (ng/mol) (log of the values); Block 2 No. oocytes; No. Embryos; No. Embryos for ET; Block 3 - Number Grade 1 Embryos; No. Grade 2 Embryos; Block 4 - No. Embryos Grade 1 (6 or more cells) and the final block included Age.

The baseline model indicated $68 \%$ predictability for the control group and a $64 \%$ for the study group. Membership of the study group had a slightly higher starting probability of being BCHG positive as $\operatorname{Exp}(B)=0.56$ (study) and $\operatorname{Exp}(B)=0.46$ (control) [Table 8]. 
Table 8: Baseline model indicating probabilities for study and control group.

\begin{tabular}{|c|c|c|c|c|}
\hline \multirow{5}{*}{ Base Model } & & Control & Study & \\
\hline & -2 Log likelihood & $101.203^{\mathrm{a}}$ & $98.764^{\mathrm{b}}$ & \\
\hline & Cox\& Snell R Square & 0.015 & 0.039 & \\
\hline & Nagelkerke R Square & 0.021 & 0.053 & \\
\hline & Overall Percentage correct & 68.293 & 64.103 & \\
\hline \multirow[t]{4}{*}{ Block 1} & -2 Log likelihood & $93.940^{\mathrm{a}}$ & $95.313^{\mathrm{a}}$ & \\
\hline & Cox\& Snell R Square & 0.098 & 0.08 & \multirow{3}{*}{$\begin{array}{l}\text { AMH (ng/mol) level relative to average (1) } \\
\qquad \text { AMH (ng/mol) (log of the values) }\end{array}$} \\
\hline & Nagelkerke R Square & 0.138 & 0.11 & \\
\hline & Overall Percentage correct & 67.073 & 65.385 & \\
\hline \multirow[t]{4}{*}{ Block 2} & -2 Log likelihood & $91.956^{\mathrm{a}}$ & $94.373^{\mathrm{b}}$ & \multirow{4}{*}{$\begin{array}{c}\text { Block } 1 \text { variables and } \\
\text { No of oocytes (trnsf) } \\
\text { No of embryos (trnsf) } \\
\text { No of embryos for ET (trnsf) }\end{array}$} \\
\hline & Cox\& Snell R Square & 0.120 & 0.091 & \\
\hline & Nagelkerke R Square & 0.168 & 0.125 & \\
\hline & Overall Percentage correct & 71.951 & 66.667 & \\
\hline \multirow[t]{4}{*}{ Block 3} & -2 Log likelihood & $91.844^{\mathrm{a}}$ & $94.355^{\mathrm{b}}$ & \multirow{4}{*}{$\begin{array}{l}\text { Block } 2 \text { variables and No Grade } 1 \\
\text { Embryos (s. trnsf) } \\
\text { No Grade } 2 \text { Embryos (trnsf) }\end{array}$} \\
\hline & Cox\& Snell R Square & 0.121 & 0.092 & \\
\hline & Nagelkerke R Square & 0.170 & 0.126 & \\
\hline & Overall Percentage correct & 73.171 & 67.949 & \\
\hline \multirow[t]{4}{*}{ Block 4} & -2 Log likelihood & $86.978^{\mathrm{a}}$ & $94.287^{\mathrm{b}}$ & \multirow{4}{*}{$\begin{array}{c}\text { Block } 3 \text { variables and No. } \\
\text { Embryos Grade } 1 \text { ( } 6 \text { or more } \\
\text { cells) (s. transf) }\end{array}$} \\
\hline & Cox\& Snell R Square & 0.172 & 0.092 & \\
\hline & Nagelkerke R Square & 0.241 & 0.127 & \\
\hline & Overall Percentage correct & 79.268 & 69.231 & \\
\hline
\end{tabular}

The full model containing all predictors was statistically insignificant, $\chi 2(9, \mathrm{~N}=82)=15.463 \mathrm{p}=0.079$ (control) and $\chi 2(9$, $\mathrm{N}=82$ ) = 7.554; $\mathrm{p}=0.58$ ), indicating that the model was able to distinguish between those who fell pregnant in the control group better than in the study group as the data had a better fit for the control group. The Hosmer and Lemeshow test were not significant for both stages (control and study), indicating some degree of fit as $\chi 2(9, \mathrm{~N}=82)=8.27 \mathrm{p}=0.408$ (control) and $\chi 2(9, \mathrm{~N}=82)=8.370$; $\mathrm{p}=0.398$. The model as a whole explained between $17 \%$ (Cox and Snell R square) and 24\% (Nagelkerke R square) of the variance between pregnancy status for the control group and correctly classified $79 \%$ of the cases. It presented far less predictive power for the study group where 9\% (Cox and Snell R square) and 12\% (Nagelkerke R square) of the variance between pregnancy status for the study group was explain and a lower $69 \%$ of the cases were correctly classified.
Of great interest is the lack of predictive power of the variables in the equation for the study group while three had predictive power for the control group. These were as follows: number of embryos extracted, number of embryos for ET and Age. The number of embryos extracted has a negative $\mathrm{B}$, as does age, indicating that higher values on both these variables decrease the probability of a positive BCGH. Number of embryos for ET has a positive B value; hence this is positively associated with pregnancy. The $\operatorname{Exp}(\mathrm{B})$, or odds ratio, must be interpreted in terms of the transformed values rather than the original values. An increase in one Embryo for ET leads to a threefold (3.5) increase in probability for a positive BHCG. An increase in one unit of age is associated with a decline in the odds of 0.85 for pregnancy. Similarly, an increase of one embryo extracted is associated with a decline in the odds of 0.6 for a positive result.

Table 9: Variable in the Equation.

\begin{tabular}{|c|c|c|c|c|c|c|c|c|}
\hline \multicolumn{9}{|c|}{ Variables in the Equation } \\
\hline
\end{tabular}




\begin{tabular}{|c|c|c|c|c|c|c|c|c|}
\hline & No. Grade 2 Embryos (s. transformed) & -58.561 & 34.519 & 2.878 & 1 & 0.09 & 0.00 \\
\hline & & $\begin{array}{c}\text { No. Embryos Grade 1 (6 or more cells) (s. trans- } \\
\text { formed) }\end{array}$ & 11.216 & 20.475 & 0.300 & 1 & 0.584 & 74291.068 \\
\hline & AGE & -.154 & 0.75 & 4.185 & 1 & 0.41 & 0.857 \\
\hline & & Constant & 3.097 & 11.633 & 0.071 & 1 & 0.079 & 0.045 \\
\hline Study & Step 1 & AMH (ng/mol) level relative to average (1) & 0.11 & 0.807 & 0.019 & 1 & 0.892 & 0.896 \\
\hline & & AMH (ng/mol) (log of the values) & 1.204 & 0.965 & 1.558 & 1 & 0.212 & 3.334 \\
\hline & & No. of oocytes (s. transformed) & 2.459 & 17.643 & 0.019 & 1 & 0.889 & 0.086 \\
\hline & & No. of Embryos (s. transformed) & 22.156 & 21.966 & 1.017 & 1 & 0.313 & 0 \\
\hline & No. of Embryos for ET (s. transformed) & 23.664 & 40.582 & 0.34 & 1 & 0.56 & $1.893 \mathrm{E}+10$ \\
\hline & No. Grade 1 Embryos (s. transformed) & 27.057 & 35.056 & 0.596 & 1 & 0.44 & $5.633 \mathrm{E}+11$ \\
\hline & No. Grade 2 Embryos (s. transformed) & 13.742 & 36.789 & 0.14 & 1 & 0.709 & 928880.339 \\
\hline & & No. Embryos Grade 1 (6 or more cells) (s. trans- & 2.184 & 24.026 & 0.008 & 1 & 0.928 & 0.113 \\
\hline & formed) & AGE & 0.019 & 0.071 & 0.068 & 1 & 0.794 & 0.982 \\
\hline & & Constant & 11.716 & 12.857 & 0.839 & 1 & 0.360 & 130040.468 \\
\hline
\end{tabular}

aVariable(s) entered on step 1: AGE.

Since none of these variables held predictive power for the study group, it can be posited that the intervention overcame their influence. This is remarkable particularly as neither age, or embryo number or embryos for transfer play a significant role. Thus, the results must be explained by variables such as the intervention [Table 9].

\section{Conclusion}

There is empirical evidence that the study group exhibited more pregnancies than the control group, although this was not statistically significant at the 0.05 level, nevertheless, women under the age of 35 were statistically more likely to fall pregnant in the study group. The study group displayed an absolute but not statistically significant higher number of pregnancies for women over the age of 35 . It must also be noted that the study and control groups were not statistically equivalent on either age or AMH (ng/ mol) level. The study group was both older on average and had far lower average AMH (ng/mol), yet, despite these characteristics demarking them as poor responders, they had significantly more pregnancies than expected for those under the age of 35 and relatively, although not statistically significantly more pregnancies for those over the age of 35 (28\% versus $25 \%$ in the control condition).

Further investigation, with the use of age and AMH (ng/mol) as continuous variables, measured ante hoc, along with a series of variables measured post hoc, shed further light on the differences between the control and the treatment conditions. While the control group exhibited acceptable levels of predictability based on established marker variables of age, number of embryos and number of embryos for ET all of which were in proportion and direction found elsewhere in the literature, these requirements did not hold true for the study group. Thus, despite being statistically older and with lower AMH (ng/mol) scores, the study group had higher pregnancy rates than expected and these rates were not explained by their age or their prior AMH (ng/mol) levels. Since embryo quality and quantity were measured post hoc to treatment, it is notable that there was no statistically significant difference between the treatment and control groups on these variables. Hence, any differences that high age or low AMH (ng/mol) levels could bring to bear on embryo quantity and quality were eliminated in the treatment programme. The treatment group; whose results could not be explained adequately by the logistic regression even though those of the control group were, have potentially overcome the disadvantages that low $\mathrm{AMH}(\mathrm{ng} / \mathrm{mol})$ and increased age present.

\section{Acknowledgements}

The study was conceived by Dr M.I. Cassim. Dr M.I. Cassim, Dr Y.M. Dasoo and Debrah Mthembu conducted all the clinical work for this study. Kerry Chipp conducted all the statistics and the analysis thereof. Dr T. Mohamed assisted with the literature review with the final write-up of the manuscript. All three authors contributed to the write-up of this manuscript.

\section{Conflict of Interest}

The authors report no conflicts of interest.

\section{References}

1. Surrey ES, Schoolcraft WB (200) Evaluating strategies for improving ovarian response of the poor responder undergoing assisted reproductive techniques. Fertility and Sterility 73(4): 667-676.

2. Artini PG, Ruggiero M, Uccelli A, Obino ME, Cela V (2013) Fertility management of patients with reduced ovarian reserve. Reproductive System and Sexual Disorders S5: 1-8.

3. Ubaldi F, Vaiarelli A, D’Anna R, Rienzi L (2014) Management of poor responders in IVF: Is there anything new? BioMed Research International 2014: 10

4. Hull KL, Harvey S (2001) Growth hormone: roles in female reproduction. J Endocrinol 168: 1-23.

5. Hull KL, Harvey S (2014) Growth hormone and reproduction: A review of endocrine and autocrine/paracrine interactions. Int J Endocrinol 2014: 234014.

6. de Ziegler D, Streuli I, Meldrum DR, Chapron C (2011) The value of growth hormone supplements in ART for poor ovarian responders. Fertil Steril 6(5): 1069-1076. 
7. Yovich JL, Stanger JD (2010) Growth hormone supplementation improves implantation and pregnancy productivity rates for poorprognosis patients undertaking IVF. Reprod Biomed Online 21(1): 3749.

8. Kolibianakis EM, Venetis CA, Diedrich K, Tarlatzis BC, Griesinger G (2009) Addition of growth hormone to gonadotrophins in ovarian stimulation of poor responders treated by in-vitro fertilization: a systematic review and meta-analysis. Hum Reprod Update 15(6): 613-622.

9. Weall BM, Al-Samerria S, Conceicao J, Yovich JL, Almahbobi G (2015) A direct action for $\mathrm{GH}$ in improvement of oocyte quality in poor responder patients. Reproduction 149(2): 147-154.
10. Bassiouny YA, Dakhly DMR, Bayoumi YA, Hashish NM (2016) Does the addition of growth hormone to the in vitro fertilization/ intracytoplasmic sperm injection antagonist protocol improve outcomes in poor responders? A randomized, controlled trial. Fertil Steril 105(3): 697-702.

11. Yu X, Ruan J, He L, Hu W, Xu Q, et al. (2015) Efficacy of growth hormone supplementation with gonadotrophins in vitro fertilization for poor ovarian responders: an updated meta-analysis. Int J Clin Exp Med 8(4): 4954-4967. 